\title{
Utility of C - reactive protein as a Novel Prognostic Marker in Patients with Carcinoma Prostate Undergoing Androgen Deprivation Therapy
}

\author{
Dineshan $\mathrm{K} \mathrm{M}^{1}$ AdityaShenoy ${ }^{2}$ Felix Cardoza ${ }^{3}$ \\ ${ }^{1}$ Additional ProfessorDepartment Of Urology, Government Medical College, Kozhikode, Kerala. \\ ${ }^{2}$, Resident,Department Of Urology, Government Medical College, Kozhikode, Kerala.. \\ ${ }^{3}$ Professor \&Head Of Department, Department Of Urology, Government Medical College, Kozhikode, Kerala.
}

\begin{abstract}
Introduction and objectives: There is increasing recognition that systemic inflammation is associated with progression and reduced survival of prostate cancer patients. C-reactive protein (CRP) is an inflammatory marker that has been evaluated as a reflection of the same. Our aim was to study the prognostic implications of pre-treatment CRP values with regard to PSA response, disease progression and short term survival in patients with carcinoma prostate undergoing androgen deprivation therapy (ADT).

Patients and methods: 86 patients with prostate cancer were subjected to measurement of CRP levels at time of diagnosis.PSA levels, Gleason score, haemoglobin, S.albumin were determined for all patients. Patients were grouped according to age, Gleason grade, PSA levels and presence of bone metastatic disease. They were followed up prospectively at preset regular intervals post ADT with relevant investigations for 12 months.

Results: CRP values were significantly elevated in patients with higher PSA (1.78 vs 9.81 for PSA /> 20ng/dl), bone metastases (18.68 vs 6.06 for no mets), and higher gleason grade.Quartiles of CRP were: 0-2.0, 2.1-6.9, 7.0-15.5, and15.6-78 mg/L On follow up, CRP was a predictive of PSA response (achieved in > $80 \%$ for patients with lowest CRP quartile compared to response in $<25 \%$ in highest quartile). In the cohort of patients with metastatic disease- CRP, low albumin levels, gleason score were prognostic factors for short term survival at 1 year. On regression analysis CRP remained an independent prognostic factor HR 1.49 inCRP $(p=0.023)$.

Conclusion: We have found that rise in CRP level is significantly associated with an aggressive tumor phenotype at the time of diagnosis.CRP can be predictive of PSA response to therapy. Also, CRP is a costeffective test that can be used as one of the markers to risk-stratify men with advanced prostate cancer.
\end{abstract}

Keywords: CRP, carcinoma prostate, $P S A$ response

\section{Introduction}

Survival in patients with prostate cancer $(\mathrm{CaP})$ has improved in recent years but prognosis remains poorly understood.Considerable effort has gone into identifying genetic/immunological biomarkers for cancer outcomes. However, these remain time consuming and not practical within routine clinical practice.Prostate cancer and Systemic InflammationThere is increasing recognition that systemic inflammation is associated with progression and reduced survival of prostate cancer patients ${ }^{1}$.Systemic inflammatory response, as evidenced by an elevated C-reactive protein (CRP), has been shown to be independently associated with poor prognosis in localized and metastatic prostate cancer ${ }^{2}$. Prostatic inflammatory atrophy, a candidate early preneoplastic lesion, is associated with a prominent inflammatory component.Inflammatory $\mathrm{T}$-cell infiltrates accompany prostate cancer.

\section{C reactive Protein}

(CRP) is an acute-phase protein produced in the liver in response to stimulation by cytokines (IL-6, IL1 , TNF). One explanation for why CRP may identify individuals at increased risk of cancer lies in its association with chronic, mostly subclinical inflammation. The kinetic of CRP is not affected by its physiological function. The momentary plasma level therefore is anexclusive indicator of the synthesis rate and thus the inflammation.High serum levels of CRP indicates poor prognosis in prostate cancer, and it is observed with the existence of bone metastasis, raised PSA and higher gleason grade ${ }^{3}$

\section{Chronic Inflammation}



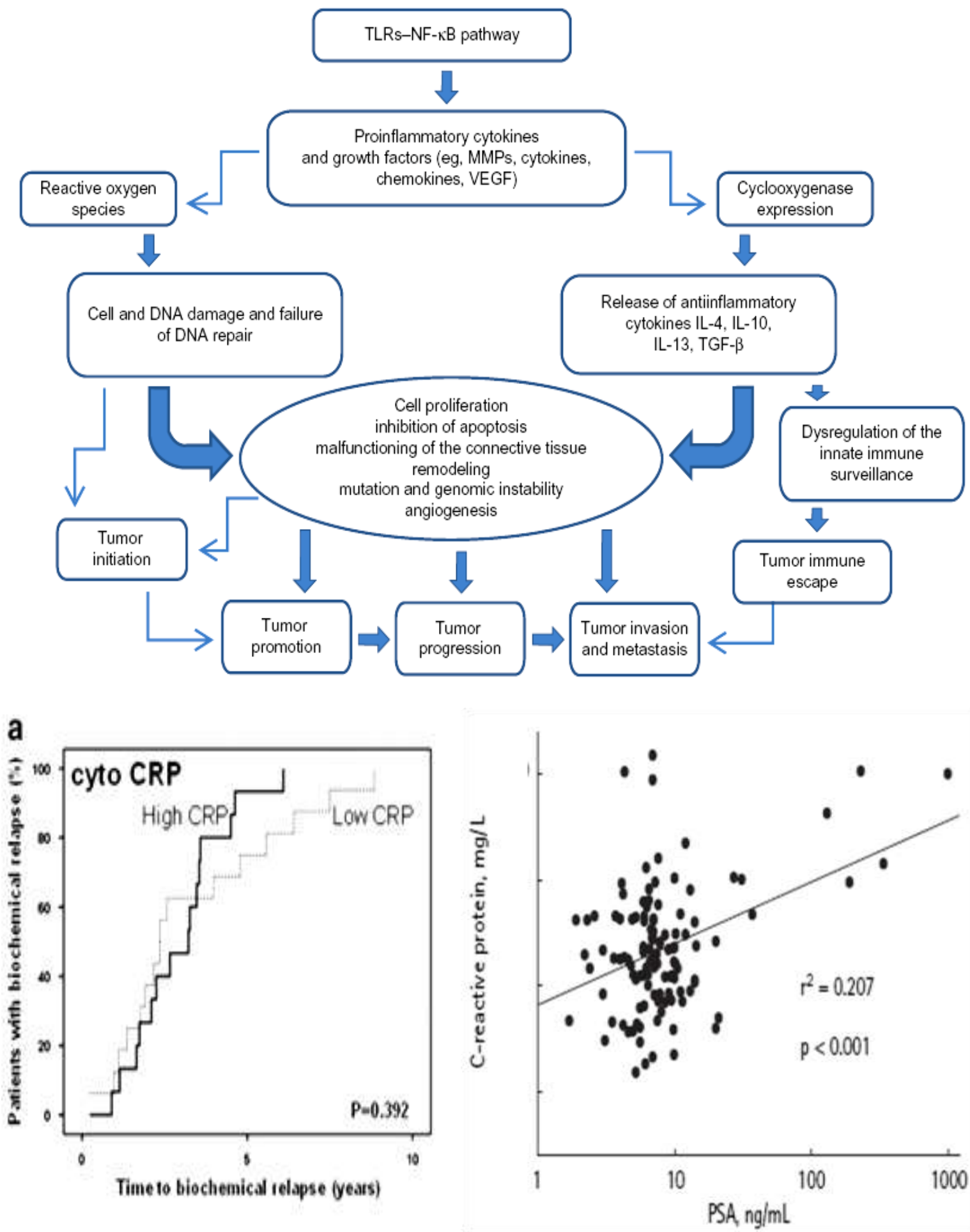

Nomograms based on clinical parameters: 


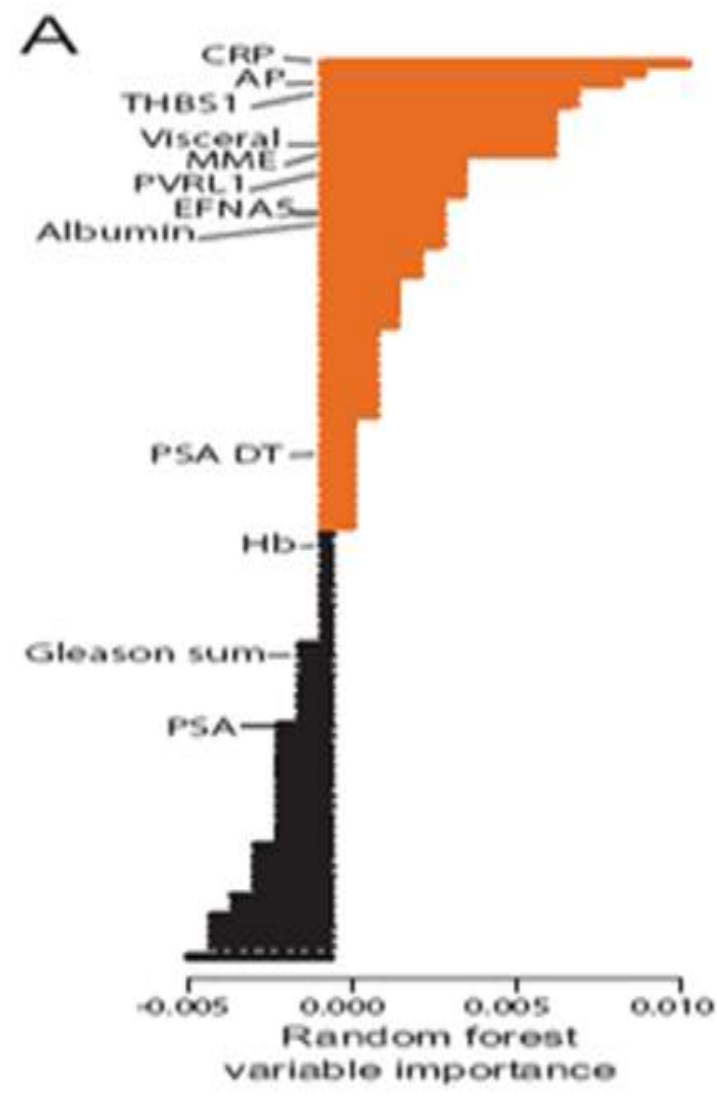

Serum lactate dekydrogenase(LDH)

Prostate-specific antigen (PSA)

Alkalme phosphatase (AP)

Gleason score

Performance status (PS)

Hemoglobim level (Hb), are used to assess proguosis

\section{Objectives}

To examine distribution of CRP values across tumor phenotypes stratified by - gleason grade, bone metastases, PSA level at the time of diagnosis.To study the prognostic implications of pre-treatment CRP values with regard to PSA response, disease progression and short term survival in patients with carcinoma prostate undergoing androgen deprivation therapy (ADT).

\section{Patients and methods}

Prospective study Jan 2012 - June 2013, Department of urology, Govt. medical collegekozhikode 86 patients with prostate cancer were subjected to quantitative measurement of CRP levels at time of diagnosis.CRP > 4mg/l considered positive. PSA levels, Gleason score and values of relevant serum biochemistries were determined for all patients. Patients underwent ADT by orchidectomy and were followed up every 3 months with examination and necessary investigations including PSA for a period of one year.Patients were assessed for response to treatment, development of metastatic disease.

Exclusion Criteria - Patients with incomplete blood parameters, active infection, h/o UTI or any febrile illness in past one month, total leukocyte count $<10,000$, CRP $>100 \mathrm{mg} / \mathrm{l}$ to avoid false positive results.

\section{Statistical Analysis}

Analysis was carried out using the statistics package SPSS (chicago IL, US)

\section{Population Characteristics}

\section{Results}

\section{Means:}

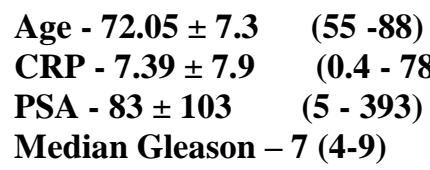

Age - $72.05 \pm 7.3$

CRP - 7.39 \pm 7.9

PSA - $83 \pm 103$

$(5-393)$

Median Gleason - 7 (4-9) 


\section{Figure 1}

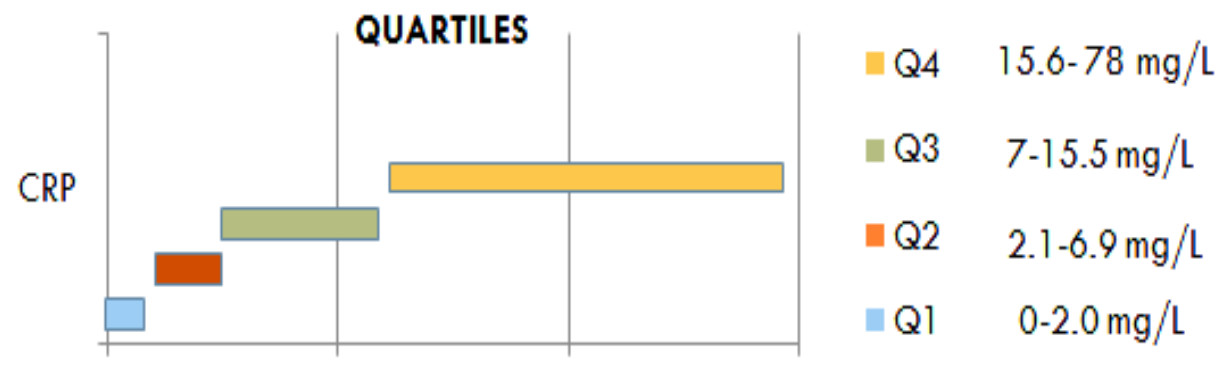

0

80

Figure 2

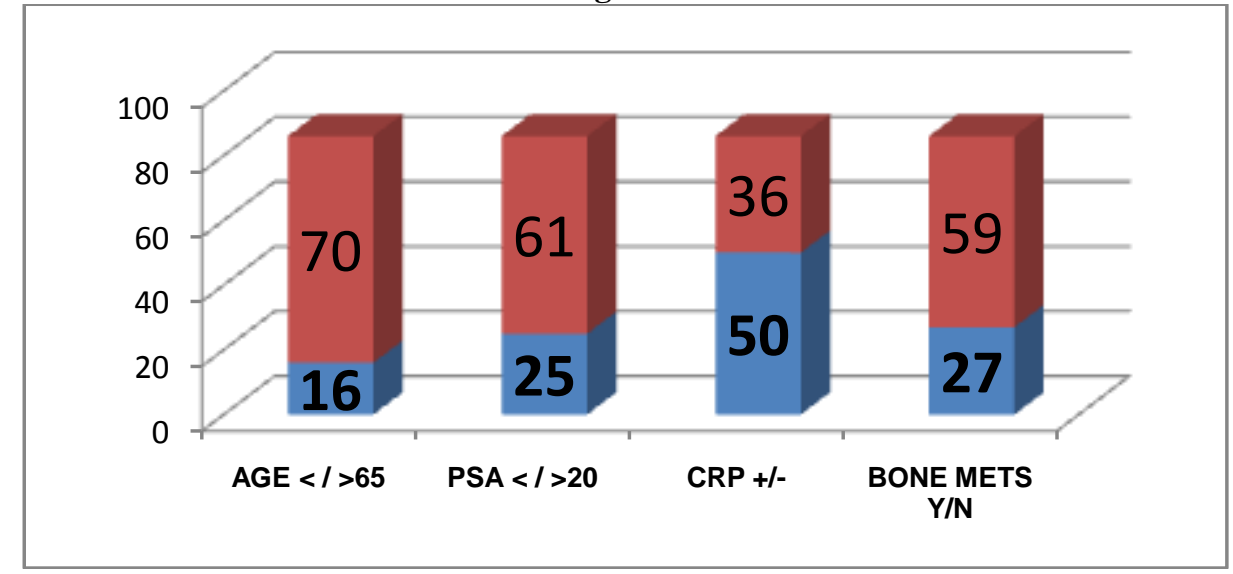

CRP was positive in $58 \%$

PSA was more than $20 \mathrm{ng} / \mathrm{dl}$ in $70.9 \%$

Bone Mets were present in $31.3 \%$

Correlation between CRP and PSA values were significant at 0.01level.

Figure 3

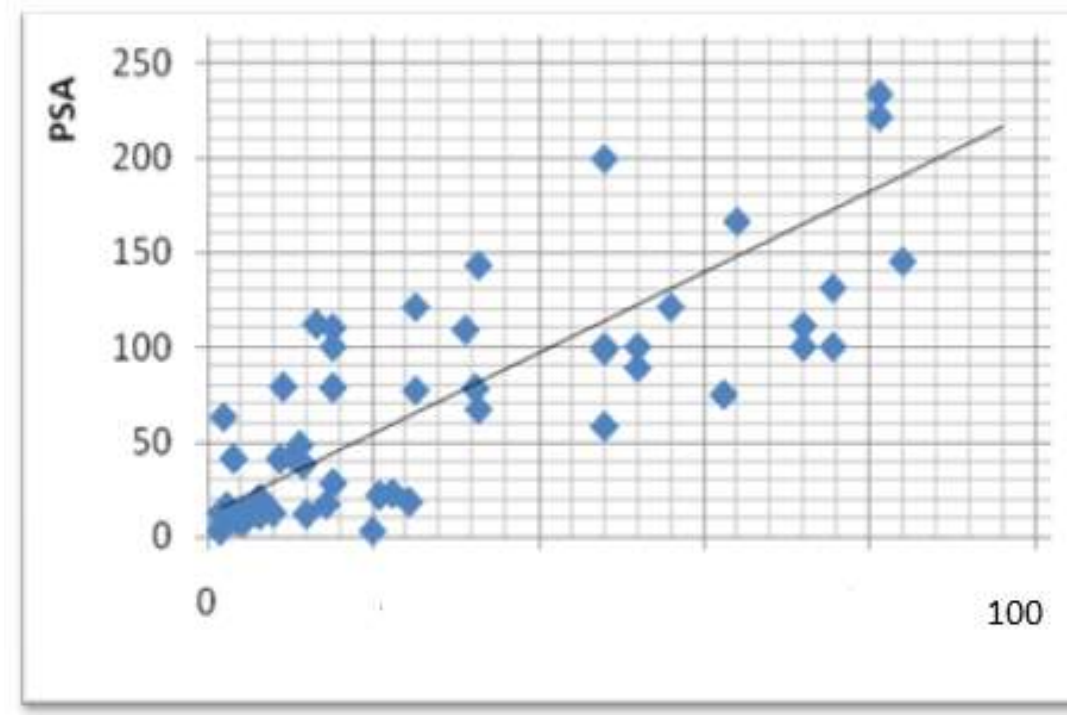

CRP was significantly elevated in patients with PSA $>20$ 
Table 1

\begin{tabular}{|l|l|l|l|l|}
\hline T Test & PSA & N & Mean & P \\
\hline \multirow{3}{*}{ CRP } & $<20$ & 25 & 1.78 & \multirow{2}{*}{0.012} \\
\cline { 2 - 4 } & $>20$ & 61 & $\mathbf{9 . 8 1}$ & \\
\hline
\end{tabular}

CRP vs Bone mets

Table 2

\begin{tabular}{|l|l|l|l|l|}
\hline T Test & PSA & N & Mean & P \\
\hline \multirow{2}{*}{ CRP } & BONE MET & 27 & $\mathbf{1 8 . 6 8}$ & \multirow{2}{*}{$\mathbf{0 . 0 0 1}$} \\
\cline { 2 - 4 } & NO METS & 59 & 6.02 & \\
\hline
\end{tabular}

Crp was 3 times on average elevated in the cohort with bone Metastasis Crpvs Gleason Grade

Table 3

Post hoc - Bonnenferroni

\begin{tabular}{|c|c|c|c|c|}
\hline ANOVA & PSA & & $\mathbf{N}$ & Mean \\
\hline \multirow{3}{*}{ CRP } & \multirow{3}{*}{ 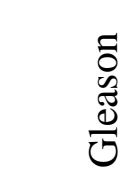 } & $2-6$ & 22 & 2.51 \\
\hline & & 7 & 36 & 5.72 \\
\hline & & $8-10$ & 28 & 14.2 \\
\hline
\end{tabular}

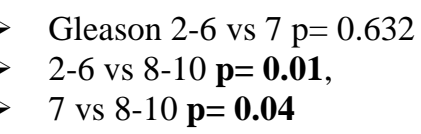

Logistic regression Analysis:Odds for Bone Metastasis

Table 4

\begin{tabular}{|l|l|l|}
\hline Variable & Odds ratio & $\mathbf{9 5 \%}$ CI \\
\hline CRP & 1.8161 & 0.919 to 3.217 \\
\hline GLS & 2.9365 & 0.488 to 17.646 \\
\hline PSA & 1.6077 & 0.974 to 2.942 \\
\hline
\end{tabular}

CRP vs Serum Parameters

Table 5

\begin{tabular}{|l|l|l|l|l|}
\hline CRP & Hb & ALB & ALP & ESR \\
\hline$<4(36)$ & 11.86 & 3.37 & 119.7 & 51 \\
\hline$>4(50)$ & 11.95 & 3.03 & 165.8 & 35 \\
\hline P Value & 0.84 & $\mathbf{0 . 0 1 1}$ & $\mathbf{0 . 0 4}$ & 0.24 \\
\hline
\end{tabular}

Association of increased ALP and decreased albumin in patients with positive CRP reflected the systemic inflammatory state.

\section{Post ADT follow up and Analysis}

Patients underwent orchidectomy and selective use of bicalutamidetherapy.PSA was determined at the end of one month and then 3 monthly thereafter.PSAresponse was defined as decrease in PSA value by atleast $50 \%$ at end of first month.Proportion of patients alive at end of one year was noted. 


\section{PSA response}

FIGURE 4
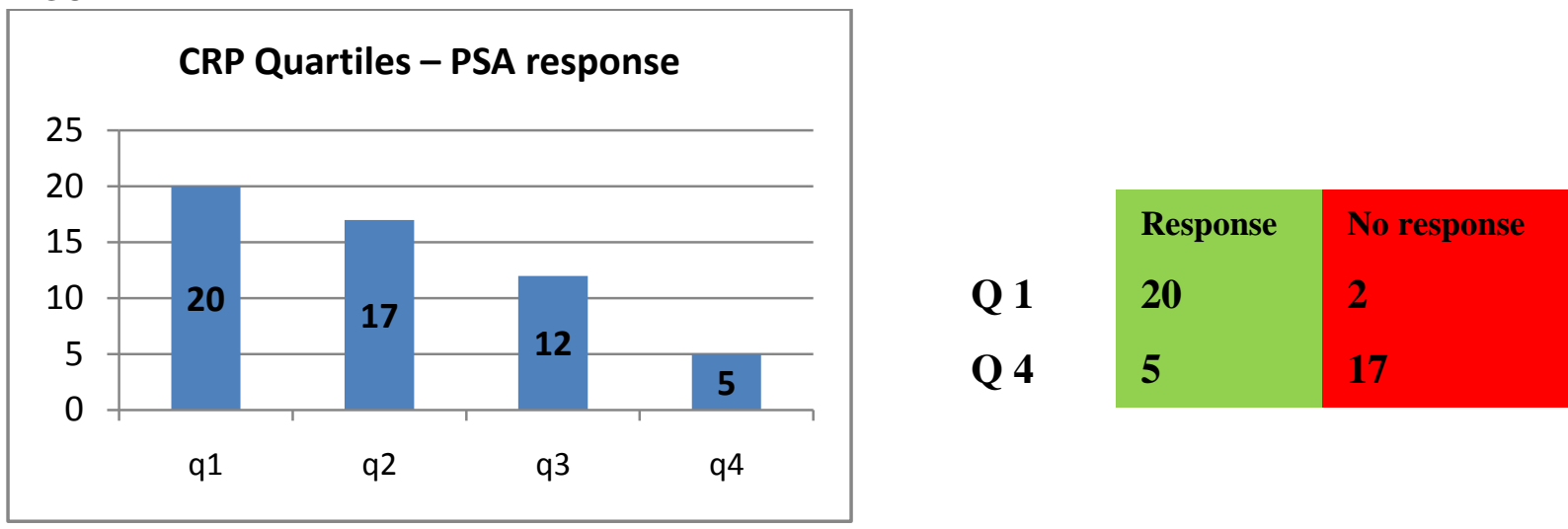

Figure 5

$\mathbf{9 0 \%}$ of patients in lowest CRP quartile achieved a response compared to only $\mathbf{2 2 \%}$ in highest quartile.Fishers Exact test $P$ value $<0.001$. Cohort of Patients with Metastasis.Comparison of means among survivors and non survivors.

Figure 6

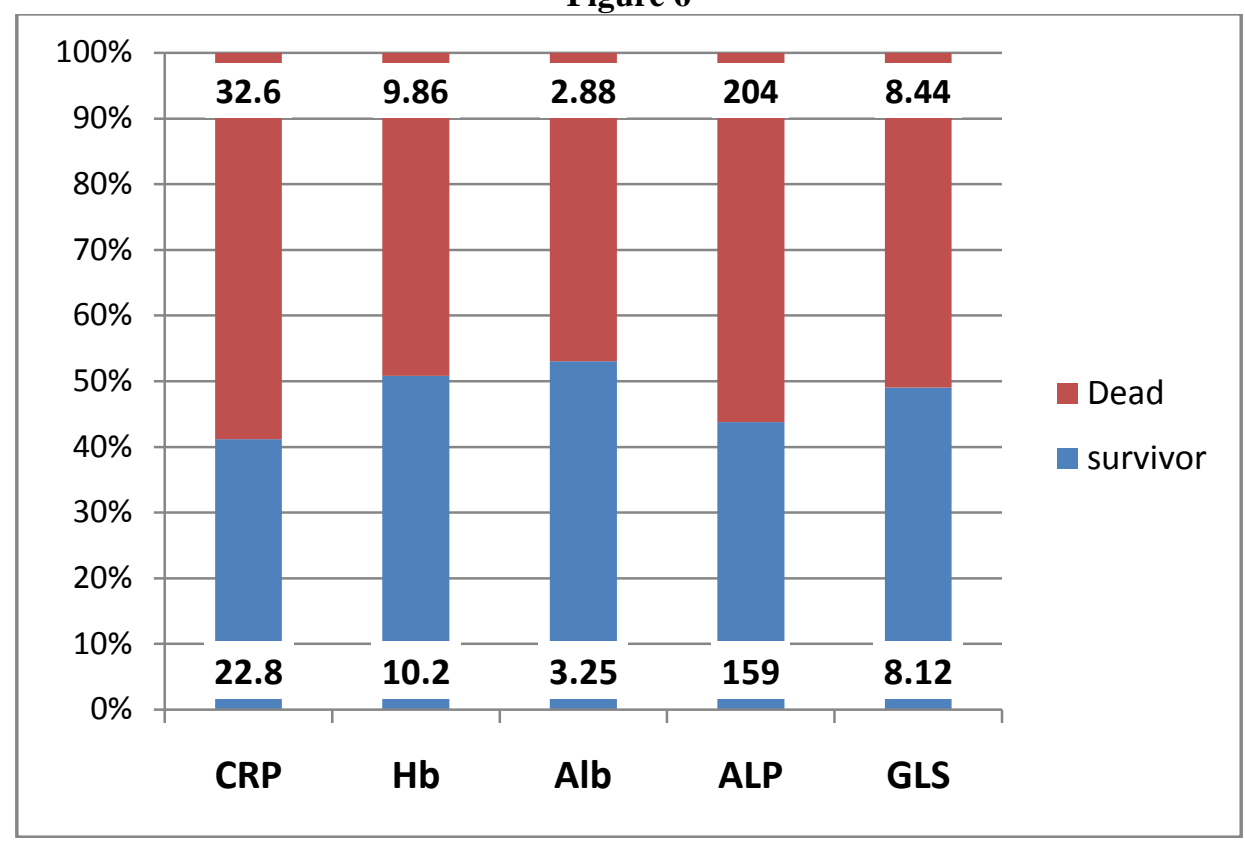

Out of 27 patients with mets 9 had died at the end of one year.

Comparison of survival curves- Patients with Metastatic disease

Figure 9 


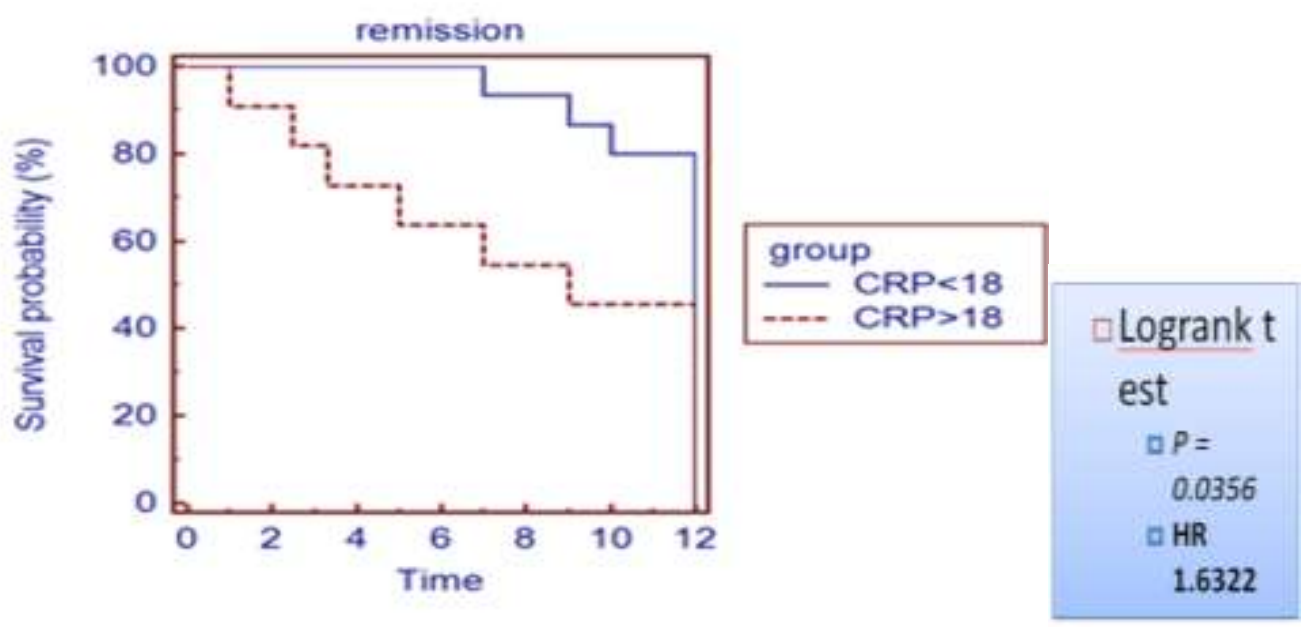

Table 6

Mean and median survival

\begin{tabular}{|l|r|r|r|r|}
\hline Factor & Mean & SE & $95 \%$ Cl for the mean & Median \\
\hline CRP $<18$ & 11.333 & 0.373 & 10.602 to 12.065 & 12.000 \\
\hline CRP $>18$ & 7.982 & 1.261 & 5.511 to 10.453 & 9.000 \\
\hline Overall & 9.915 & 0.613 & 8.714 to 11.117 & 12.000 \\
\hline
\end{tabular}

\section{Limitations}

A sample size is modest for an analysis of prognostic marker in this patient population.The study was an exploratory and pilot effort and the results should be confirmed in a large cohort.Parent markers driving CRP like IL-6 are being currently explored

\section{Conclusions}

Higher CRP level is significantly associated with an aggressive tumor phenotype at the time of diagnosis in Carcinoma Prostate. CRP can be predictive of PSA response to therapy. CRP is a cost-effective test that can be used as one of the markers to risk-stratify men with advanced prostate cancer. CRP could prove to be a useful and readily measurable prognostic marker that could aid in clinical decision-making, patient counseling, and clinical trial design and interpretation.

\section{Reference}

[1]. Platz E. Epidemiology of inflammation and prostate cancer.J Urol2004;171: S36-40

[2]. Elsberger B, Edwards J: Presence of tumoural C-reactive protein correlates with progressive prostate cancer. Prostate Cancer Prostatic Dis 2011, 14:122-128.

[3]. Bastian PJ, et al. Prostate carcinogenesis and inflammation: emerging insights. Carcinogenesis.2005;26(7): 1170-1181.

[4]. Lehrer S, C-reactive protein is significantly association with prostate-specific antigen and metastatic disease in prostate cancer. BJU International. 2005;95:961-62.

[5]. Small EJ, et al. Nomogram for overall survival of patients with progressive metastatic prostate cancer after castration. J ClinOncol 2012;20:3972-82. 